\title{
Solar wind flow near a reconnection site at the dayside magnetopause: development of an existing three-dimensional model
}

\author{
LARS G. WESTERBERG and H. O. Å ERSTEDT \\ Division of Fluid Mechanics, Luleå University of Technology, SE-971 87, Luleå, Sweden \\ (lars-goran.westerberg@itu.se)
}

(Received 23 October 2006)

\begin{abstract}
An improvement of an existing three-dimensional analytical model describing the solar wind flow near a reconnection site at the dayside magnetopause is reported. Introducing an arbitrary orientation of the reconnection line, general solutions for the plasma velocity and magnetic field during the transition of the magnetopause are presented, together with the development of the magnetopause transition layer away from the reconnection site.
\end{abstract}

\section{Introduction}

Recently, Westerberg and Åkerstedt (2006) made a three-dimensional analysis covering the solar wind flow near a reconnection site at the terrestrial magnetopause. The objective of their study was to obtain a description of the velocity and magnetic field structure during the transition from the magnetosheath to the magnetosphere, together with the development of the magnetopause transition layer separating magnetosheath plasma from magnetosphere plasma. However, their model covers only a special case where the reconnection line is aligned with the $y$-axis. The main objective of the present paper is to present an extension of the previous analysis by introducing an orientation of the reconnection line that is arbitrary in the $y z$-plane (Fig. 1(b)), and solve the governing equations for a new set of coordinates.

Considering a three-dimensional flow we let

$$
\begin{aligned}
& \mathbf{u}=u_{x} \hat{\mathbf{x}}+u_{y} \hat{\mathbf{y}}+u_{z} \hat{\mathbf{z}} \\
& \mathbf{B}=B_{x} \hat{\mathbf{x}}+B_{y} \hat{\mathbf{y}}+B_{z} \hat{\mathbf{z}}
\end{aligned}
$$

where $\hat{\mathbf{x}}, \hat{\mathbf{y}}, \hat{\mathbf{z}}$ represent a local plane coordinate system close to the reconnection line, with $\hat{\mathbf{x}}$ pointing in the normal direction of the magnetopause, $\hat{\mathbf{z}}$ pointing in the tangential direction, and $\hat{\mathbf{y}}$ completing the right-hand system. The coordinates $x, y, z$ are thus identical to the local plane magnetopause coordinates $L, M, N$ (Russell and Elphic 1978), such that $z=L, y=M$, and $x=N$ (see Fig. 1(a)). 

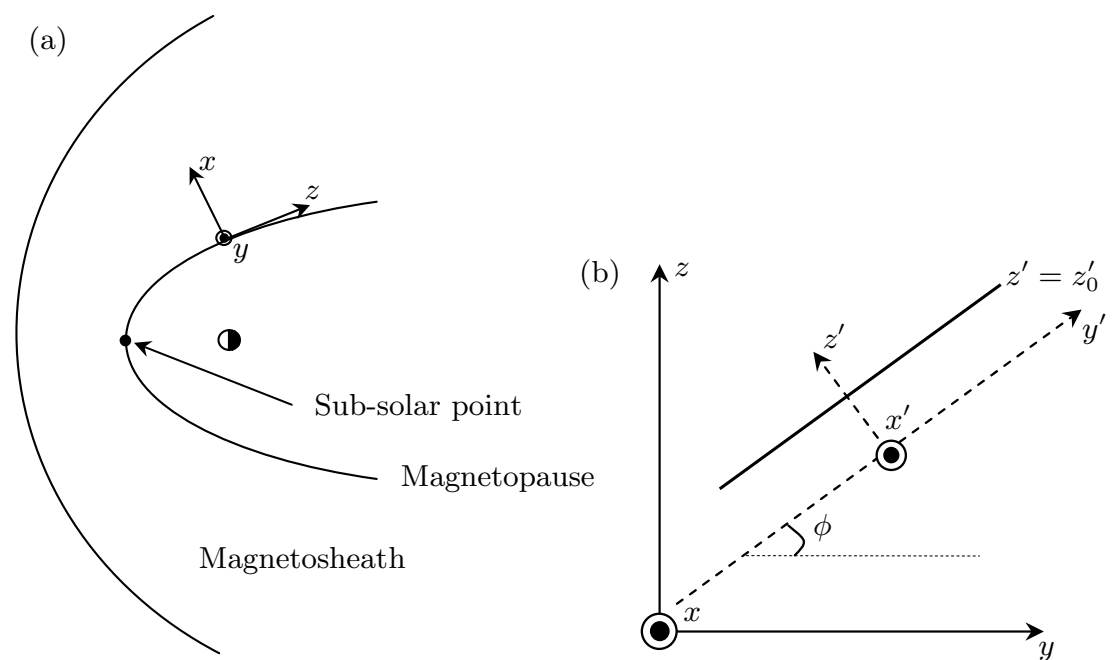

Figure 1. Curvilinear coordinate system and orientation of the reconnection line. (a) The curvilinear coordinate system with conventional labels for the coordinate axis, with $x$ pointing in a normal direction to the magnetopause surface, $z$ in the corresponding tangential direction, and $y$ completing the right-hand system. It is identical to the magnetopause boundary coordinates $(L, M, N)$ (Russell and Elphic 1978), with $z=L, y=M$, and $x=N$. (b) The orientation of the reconnection line with a new coordinate system, where $z^{\prime}$ is normal to the reconnection line, $y^{\prime}$ is aligned with the reconnection line, and $x^{\prime}=x$.

The dimensionless equations describing the plasma properties north and south of a reconnection line are (Westerberg and Åkerstedt 2006)

$$
\begin{aligned}
\hat{\mathbf{y}}: \quad \alpha & \frac{\partial^{2} u_{y}^{(0)}}{\partial \xi^{2}}+2 \xi\left(\frac{\partial U_{y}}{\partial y}+\frac{\partial U_{z}}{\partial z}\right) \frac{\partial u_{y}^{(0)}}{\partial \xi}+u_{y}^{(0)} \frac{\partial U_{z}}{\partial z}-2 U_{y} \frac{\partial u_{y}^{(0)}}{\partial y} \\
& -U_{y} \frac{\partial u_{z}^{(0)}}{\partial z}-U_{y} \frac{\partial U_{y}}{\partial y}-u_{z}^{(0)} \frac{\partial U_{y}}{\partial z}-U_{z}\left(\frac{\partial u_{y}^{(0)}}{\partial z}+\frac{\partial U_{y}}{\partial z}\right)-\frac{\partial P}{\partial y}=0 \\
\hat{\mathbf{z}}: \quad \alpha & \frac{\partial^{2} u_{z}^{(0)}}{\partial \xi^{2}}+2 \xi\left(\frac{\partial U_{y}}{\partial y}+\frac{\partial U_{z}}{\partial z}\right) \frac{\partial u_{z}^{(0)}}{\partial \xi}+u_{z}^{(0)} \frac{\partial U_{y}}{\partial y}-2 U_{z} \frac{\partial u_{z}^{(0)}}{\partial z} \\
& -U_{z} \frac{\partial u_{y}^{(0)}}{\partial y}-U_{y}\left(\frac{\partial u_{z}^{(0)}}{\partial y}+\frac{\partial U_{z}}{\partial y}\right)-U_{z} \frac{\partial U_{z}}{\partial z}-u_{y}^{(0)} \frac{\partial U_{z}}{\partial y}-\frac{\partial P}{\partial z}=0 .
\end{aligned}
$$

$U_{y, z}$ are the respective components of the DeHoffmann-Teller (DHT) velocity (DeHoffmann and Teller 1950; Westerberg and Åkerstedt 2006), $\alpha=\left(1+\eta_{\mathrm{d}} / \nu\right)$ where $\eta_{\mathrm{d}}$ is the magnetic diffusivity and $\nu$ the kinematic viscosity, and $u_{y, z}^{(0)}$ are the respective components of the plasma velocity with respect to the DHT frame of reference. $\xi$ is the re-scaled $x$-coordinate such that $\xi=R^{1 / 2} x$. See Westerberg and Åkerstedt (2006) for details of the analysis.

\section{Solutions}

For the solution to (1.2) and (1.3) the DHT velocities are expanded such that

$$
\begin{aligned}
& U_{y}=U_{y}^{0}, \\
& U_{z}=U_{z}^{0} .
\end{aligned}
$$


In order to match the analytical solutions with satellite data only the leadingorder term in $z$ is utilized. Matching higher-order terms requires gradients of the magnetic field and velocity field, which are not known from satellite data. The DHT velocity as it is defined in the analysis is related to the experimental value of the DHT velocity obtained through minimisation of the residual electric field (Sonnerup and Scheible 2000), such that

$$
\mathbf{U}_{\mathrm{DHT}}=\mathbf{V}_{\mathrm{DHT}}-\mathbf{V}_{\mathrm{DHT}, \mathrm{n}} .
$$

The DHT velocity to be used is thus the difference between the experimentally calculated DHT velocity and its component normal to the magnetopause boundary. Transforming to the $x^{\prime} y^{\prime} z^{\prime}$-coordinate system (Fig. 1(b)) we obtain

$$
\begin{aligned}
y^{\prime} & =y \cos (\phi)+z \sin (\phi), \\
z^{\prime} & =-y \sin (\phi)+z \cos (\phi), \\
\xi^{\prime} & =\xi \\
\frac{\partial}{\partial y} & =\cos (\phi) \frac{\partial}{\partial y^{\prime}}-\sin (\phi) \frac{\partial}{\partial z^{\prime}}, \\
\frac{\partial}{\partial z} & =\sin (\phi) \frac{\partial}{\partial y^{\prime}}+\cos (\phi) \frac{\partial}{\partial z^{\prime}} \\
\frac{\partial}{\partial \xi} & =\frac{\partial}{\partial \xi^{\prime}} .
\end{aligned}
$$

Applying (2.1) and (2.3) to (1.2) and (1.3), the equation system can be written as

$$
\begin{aligned}
& {\left[\begin{array}{cc}
2 U_{z}^{0} \cos (\phi)-U_{y}^{0} \sin (\phi) & -U_{z}^{0} \sin (\phi) \\
U_{y}^{0} \cos (\phi) & -2 U_{y}^{0} \sin (\phi)+U_{z}^{0} \cos (\phi)
\end{array}\right] \frac{\partial}{\partial z^{\prime}}\left[\begin{array}{c}
u_{z}^{(0)} \\
u_{y}^{(0)}
\end{array}\right]} \\
& +\left[\begin{array}{cc}
2 U_{z}^{0} \sin (\phi)+U_{y}^{0} \cos (\phi) & U_{z}^{0} \cos (\phi) \\
U_{y}^{0} \sin (\phi) & 2 U_{y}^{0} \cos (\phi)+U_{z}^{0} \sin (\phi)
\end{array}\right] \frac{\partial}{\partial y^{\prime}}\left[\begin{array}{c}
u_{z}^{(0)} \\
u_{y}^{(0)}
\end{array}\right] \\
& =\alpha \frac{\partial^{2}}{\partial \xi^{\prime 2}}\left[\begin{array}{c}
u_{z}^{(0)} \\
u_{y}^{(0)}
\end{array}\right] .
\end{aligned}
$$

Using the Jordan matrix $(\mathbf{J})$ we can express the system as

$$
\frac{\partial \mathbf{u}}{\partial z}+\mathbf{P}^{-1} \mathbf{J P} \frac{\partial \mathbf{u}}{\partial y}=\alpha \frac{\partial^{2}}{\partial \xi^{\prime 2}} \mathbf{A}^{-1} \mathbf{u}
$$

where

$$
\mathbf{J}=\left[\begin{array}{cc}
\frac{U_{y}^{0} \cos (\phi)+U_{z}^{0} \sin (\phi)}{U_{z}^{0} \cos (\phi)-U_{y}^{0} \sin (\phi)} & 1 \\
0 & \frac{U_{y}^{0} \cos (\phi)+U_{z}^{0} \sin (\phi)}{U_{z}^{0} \cos (\phi)-U_{y}^{0} \sin (\phi)}
\end{array}\right]
$$




$$
\begin{gathered}
\mathbf{P}=\left[\begin{array}{cc}
0 & \frac{2\left(2 U_{z}^{0} U_{y}^{0} \sin (\phi) \cos (\phi)-U_{z}^{0^{2}} \cos (\phi)^{2}-U_{y}^{0^{2}}+U_{y}^{0^{2}} \cos (\phi)^{2}\right)}{U_{y}^{0^{2}}} \\
1 & -\frac{U_{z}^{0}}{U_{y}^{0}}
\end{array}\right], \\
\mathbf{A}=\left[\begin{array}{cc}
2 U_{z}^{0} \cos (\phi)-U_{y}^{0} \sin (\phi) & -U_{z}^{0} \sin (\phi) \\
U_{y}^{0} \cos (\phi) & -2 U_{y}^{0} \sin (\phi)+U_{z}^{0} \cos (\phi)
\end{array}\right], \\
\mathbf{u}=\left[\begin{array}{c}
u_{z}^{(0)} \\
u_{y}^{(0)}
\end{array}\right] .
\end{gathered}
$$

Introducing $\mathbf{V}=\mathbf{P u}$ such that

$$
\begin{aligned}
V_{1} & =u_{y}^{(0)} \frac{2\left(2 U_{z}^{0} U_{y}^{0} \sin (\phi) \cos (\phi)-U_{z}^{0^{2}} \cos (\phi)^{2}-U_{y}^{0^{2}}+U_{y}^{0^{2}} \cos (\phi)^{2}\right)}{U_{y}^{0^{2}}}, \\
V_{2} & =u_{z}^{(0)}-u_{y}^{(0)} \frac{U_{z}^{0}}{U_{y}^{0}},
\end{aligned}
$$

gives

$$
\frac{\partial \mathbf{V}}{\partial z^{\prime}}+\mathbf{J} \frac{\partial \mathbf{V}}{\partial y^{\prime}}=\frac{\partial^{2}}{\partial \xi^{\prime 2}} \mathbf{P A}^{-1} \mathbf{u}
$$

resulting in

$$
\begin{aligned}
\frac{\partial V_{1}}{\partial z^{\prime}}+a \frac{\partial V_{1}}{\partial y^{\prime}}+\frac{\partial V_{2}}{\partial y^{\prime}} & =\alpha\left(\frac{\cos (\phi)}{U_{y}^{0}} \frac{\partial^{2} V_{2}}{\partial \xi^{\prime 2}}+\frac{1}{2 b} \frac{\partial^{2} V_{1}}{\partial \xi^{\prime 2}}\right), \\
\frac{\partial V_{2}}{\partial z^{\prime}}+a \frac{\partial V_{2}}{\partial y^{\prime}} & =\frac{\alpha}{b} \frac{\partial^{2} V_{2}}{\partial \xi^{\prime 2}} .
\end{aligned}
$$

Here

$$
\begin{aligned}
& a=\frac{U_{y}^{0} \cos (\phi)+U_{z}^{0} \sin (\phi)}{U_{z}^{0} \cos (\phi)-U_{y}^{0} \sin (\phi)}, \\
& b=U_{z}^{0} \cos (\phi)-U_{y}^{0} \sin (\phi) .
\end{aligned}
$$

Having (2.13) in parabolical form, we seek to find a self-similar solution of the form

$$
\begin{aligned}
\eta & =\xi^{\prime} g\left(Z^{\prime}\right)=\frac{\xi^{\prime}}{\delta\left(Z^{\prime}\right)}, \\
Z^{\prime} & =z^{\prime}-z_{0}^{\prime},
\end{aligned}
$$

where $\delta\left(Z^{\prime}\right)$ is the transition layer thickness as a function of the distance from the reconnection line. This results in a new set of partial derivatives

$$
\frac{\partial^{2}}{\partial \xi^{\prime 2}}=\frac{1}{\delta^{2}} \frac{\partial^{2}}{\partial \eta^{2}},
$$




$$
\begin{aligned}
\frac{\partial}{\partial y^{\prime}} & =0 \\
\frac{\partial}{\partial Z^{\prime}} & =\frac{\partial}{\partial Z^{\prime}}-\eta \frac{\delta^{\prime}}{\delta} \frac{\partial}{\partial \eta} .
\end{aligned}
$$

Equation (2.13) can then be written in terms of the new variables as

$$
\begin{aligned}
& \frac{\partial V_{1}}{\partial z^{\prime}}-\eta \frac{\delta^{\prime}}{\delta} \frac{\partial V_{2}}{\partial \eta}=\frac{\alpha}{\delta^{2}}\left(\frac{\cos (\phi)}{U_{y}^{0}} \frac{\partial^{2} V_{2}}{\partial \eta^{2}}+\frac{1}{2 b} \frac{\partial^{2} V_{1}}{\partial \eta^{2}}\right) \\
& \frac{\partial V_{2}}{\partial z^{\prime}}+\eta \frac{\delta^{\prime}}{\delta} \frac{\partial V_{2}}{\partial \eta}=\frac{\alpha}{b} \frac{\partial^{2} V_{2}}{\partial \xi^{\prime 2}} .
\end{aligned}
$$

For the solution to (2.17) we expand $V_{1}$ and $V_{2}$ such that

$$
\begin{aligned}
& V_{1}=V_{10}(\eta)+Z^{\prime} V_{11}(\eta), \\
& V_{2}=V_{20}(\eta)+Z^{\prime} V_{21}(\eta) .
\end{aligned}
$$

Applying (2.18) in (2.17) gives to lowest order

$$
\begin{aligned}
V_{20}^{\prime \prime}+\frac{b}{\alpha} \delta^{\prime} \delta \eta V_{20}^{\prime} & =0, \\
V_{10}^{\prime \prime}+\frac{2 b}{\alpha} \delta^{\prime} \delta \eta V_{10}^{\prime} & =-\frac{2}{a} V_{20}^{\prime \prime},
\end{aligned}
$$

with solutions

$$
\begin{aligned}
V_{20}= & C_{1}+C_{2} \cdot \operatorname{erf}\left(\frac{1}{2} \sqrt{2 k_{1}} \eta\right) \\
V_{10}= & \frac{2 C_{2} \cos (\phi)}{U_{y}^{0}}\left(U_{z}^{0} \cos (\phi)-U_{y}^{0} \sin (\phi)\right) \cdot \operatorname{erf}\left(\frac{1}{2} \sqrt{2 k_{1}} \eta\right) \\
& +\frac{C_{3}}{2 \sqrt{k_{1} / \pi}} \cdot \operatorname{erf}\left(\sqrt{k_{1}} \eta\right)+C_{4},
\end{aligned}
$$

where $k_{1}=b \delta^{\prime} \delta / \alpha$ is a constant. This results in the expression for the transition layer thickness

$$
\delta\left(Z^{\prime}\right)=\sqrt{\frac{2 \alpha k_{1}}{U_{z}^{0} \cos (\phi)-U_{y}^{0} \sin (\phi)} Z^{\prime}} .
$$

In order to obtain real solutions, $k_{1}$ has to be positive. Furthermore, a condition for $\phi$ is obtained to satisfy $\left(U_{z}^{0} \cos (\phi)-U_{y}^{0} \sin (\phi)\right)>0$ when $Z^{\prime}>0$ (north of the reconnection line), and $\left(U_{z}^{0} \cos (\phi)-U_{y}^{0} \sin (\phi)\right)<0$ when $Z^{\prime}<0$ (south of the reconnection line).

Figure 2 shows an example of the development of $\mathbf{u}$ and $\mathbf{B}$ north of the reconnection site, during the transition of the magnetopause. Matching conditions from measurements made by Cluster spacecraft 4 during a magnetopause crossing on 13 January 2002. For $U_{z}^{0}=1.5, U_{y}^{0}=1$, and $k_{1}$ chosen to be equal to 2 , we obtain the condition $-90^{\circ}<\phi<56^{\circ}$. Each graph includes plots for $\phi=54^{\circ}, \phi=30^{\circ}$, and $\phi=-89^{\circ}$. 

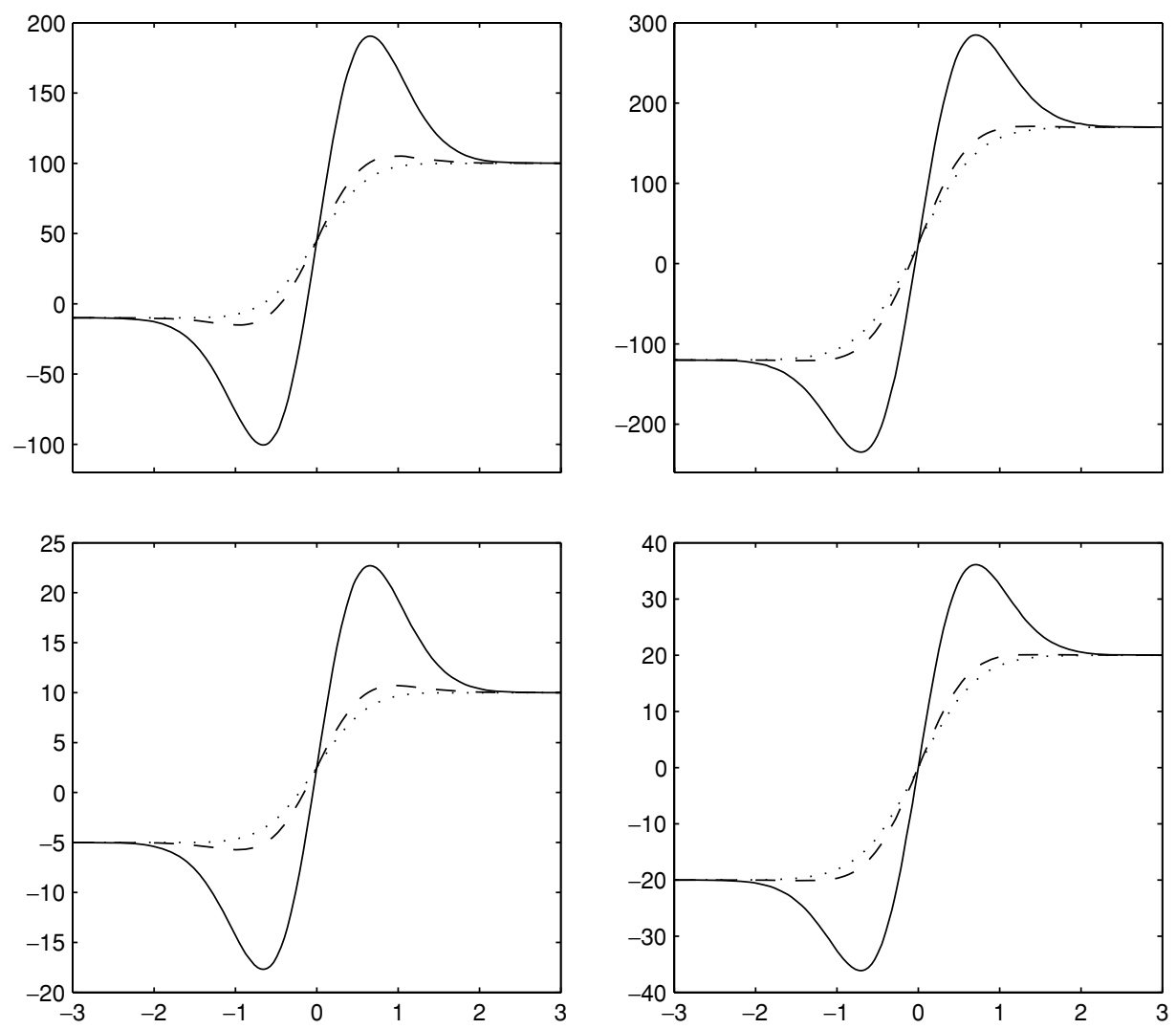

Figure 2. Example of the development north of the reconnection line for $\mathbf{u}$ and $\mathbf{B}$ during the transition of the magnetopause. The current length scale on the $x$-axis is in Earth radii $\left(R_{\mathrm{E}}\right)$. The asterisk denotes dimensional quantity. Here $U_{z}^{0}=1.5$ and $U_{y}^{0}=1$, giving the condition $-90^{\circ}<\phi<56^{\circ}$. The solid line, dashed line, and dotted line represent $\phi=54^{\circ}, \phi=30^{\circ}$, and $\phi=-89^{\circ}$, respectively.

\section{Summary}

In this paper we have introduced an orientation of the reconnection line that is not limited to being aligned with the $y$-axis, and a development of an existing 3D analytical model covering the plasma flow behaviour near a reconnection site is presented. Using satellite data gathered by the Cluster 4 spacecraft, we present a case where the reconnection line is located in the interval $-90^{\circ}<\phi<56^{\circ}$ (Fig. 2). Furthermore, it is shown that near the upper limit the effect of plasma flux pile up is significant. It is also shown that the thickness of the transition region decreases from a maximum near the lower limit of $\phi$, when approaching the upper limit.

\section{Acknowledgement}

The present research project is financed through the Swedish National Graduate School of Space Technology. 


\section{References}

DeHoffmann, F. and Teller, E. 1950 Magnetohydrodynamic shocks. Phys. Rev. Lett. 80, 692. Russell, C. T. and Elphic, R. C. 1978 Initial ISEE magnetometer results: magnetopause observations. Space Sci. Rev. 22, 681.

Sonnerup, B. U. Ö. and Scheible, M. 2000 Minimum and maximum variance analysis. In: Analysis Methods for Multi-Spacecraft Data (ed. G. Pashmann and P. W. Daly). ISSI Scientific Report.

Westerberg, L. G. and Åkerstedt, H. O. 2006 A three-dimensional analysis of the flow past an open terrestrial magnetopause. J. Plasma Physics 72(3), 359-382. 